UDC: $517.518 .15: 517.16$

45 (LXXI) No.2 (2021), 95-106

DOI: $10.37560 /$ matbil21452095m

\title{
NEW INTEGRAL INEQUALITIES FOR PREINVEX FUNCTIONS VIA CAPUTO FRACTIONAL DERIVATIVES
}

\author{
BADREDDINE MEFTAH $^{1}$ AND ARTION KASHURI ${ }^{2}$
}

\begin{abstract}
In this paper we present the analogue of Hermite-Hadamard inequality for preinvex functions via Caputo fractional derivatives. Moreover, we establish a new integral identity and derive some new trapezium type inequalities for preinvex functions via Caputo fractional derivatives.
\end{abstract}

\section{INTRODUCTION AND PRELIMINARIES}

Definition 1.1. 1$]$ A function $f: I \rightarrow \mathbb{R}$ is said to be convex, if

$$
f(t x+(1-t) y) \leq t f(x)+(1-t) f(y)
$$

holds for all $x, y \in I$ and $t \in[0,1]$.

One of the famous inequalities for the class of convex functions is the so-called Hermite-Hadamard inequality 2, 3, which can be stated as follows: For every convex function $f$ on the interval $[a, b]$ with $a<b$, we have

$$
f\left(\frac{a+b}{2}\right) \leq \frac{1}{b-a} \int_{a}^{b} f(x) d x \leq \frac{f(a)+f(b)}{2} .
$$

The above inequality has caught the attention of many mathematicians from all over the world. Since its discovery several generalizations, improvements and extensions via various classes of classical and generalized convex functions, classical and fractional variants have been introduced. For some papers connected with (1.1) interested readers are advised to consult [4, 5, 6, 7, 8, 9, 10, 11, 12, 13, and references therein.

Dragomir and Agarwal [14, established the following inequality connected with inequality (1.1).

$$
\left|\frac{f(a)+f(b)}{2}-\frac{1}{b-a} \int_{a}^{b} f(x) d x\right| \leq \frac{b-a}{8}\left(\left|f^{\prime}(a)\right|+\left|f^{\prime}(b)\right|\right) .
$$

2010 Mathematics Subject Classification. Primary: 26D10 Secondary: 26D15, 26A33, 26A51.

Key words and phrases. Hermite-Hadamard inequality; Hölder's inequality; power mean inequality; Caputo fractional derivatives; preinvex functions. 
Let us recall some basic known definitions and results.

Definition 1.2. 15] A function $f: I=[a, b] \subset[0,+\infty) \rightarrow \mathbb{R}$ is called s-convex, if

$$
f(t x+(1-t) y) \leq t^{s} f(x)+(1-t)^{s} f(y)
$$

holds for all $x, y \in I$ and $t \in[0,1]$.

Definition 1.3. [16] $A$ set $K \subseteq \mathbb{R}^{n}$ is said an invex with respect to the bifunction $\eta: K \times K \rightarrow \mathbb{R}^{n}$, if for all $x, y \in K$, we have

$$
x+t \eta(y, x) \in K .
$$

Definition 1.4. [16] A function $f: K \rightarrow \mathbb{R}$ is said to be preinvex, if

$$
f(x+t \eta(y, x)) \leq(1-t) f(x)+t f(y)
$$

holds for all $x, y \in K$ and $t \in[0,1]$.

Condition $\mathbf{C}$ [17] Let $K$ be an invex set with respect to the bifunction $\eta(\cdot, \cdot)$ then for any $a, b \in K$ and $t \in[0.1]$, we have

$$
\eta(a, a+t \eta(b, a))=-t \eta(b, a) \text { and } \eta(b, a+t \eta(b, a))=(1-t) \eta(b, a) .
$$

From Condition $\mathrm{C}$, it follows that

$$
\eta\left(a+t_{2} \eta(b, a), a+t_{1} \eta(b, a)\right)=\left(t_{2}-t_{1}\right) \eta(b, a)
$$

for any $a, b \in K$ and $t_{1}, t_{2} \in[0.1]$.

Noor [18, gave the analogue preinvex of inequality (1.1) as follows:

$$
f\left(\frac{2 a+\eta(b, a)}{2}\right) \leq \frac{1}{\eta(b, a)} \int_{a}^{a+\eta(b, a)} f(x) d x \leq \frac{f(a)+f(b)}{2} .
$$

Barani et al. [19], established the following inequality for differentiable preinvex functions.

$$
\left|\frac{f(a)+f(a+\eta(b, a))}{2}-\frac{1}{\eta(b, a)} \int_{a}^{a+\eta(b, a)} f(x) d x\right| \leq \frac{\eta(b, a)}{8}\left(\left|f^{\prime}(a)\right|+\left|f^{\prime}(b)\right|\right) .
$$

Definition 1.5. 20] Let $\alpha>0$ and $\alpha \notin\{1,2,3, \ldots\}, n=[\alpha]+1, f \in A C^{n}[a, b]$ which is the space of functions having $n^{\text {th }}$ derivatives absolutely continuous. The right-sided and left-sided Caputo fractional derivatives of order $\alpha$ are defined as follows:

and

$$
\left({ }^{c} D_{a^{+}}^{\alpha} f\right)(x)=\frac{1}{\Gamma(n-\alpha)} \int_{a}^{x}(x-t)^{n-\alpha-1} f^{(n)}(t) d t, x>a
$$

$$
\left({ }^{c} D_{b^{-}}^{\alpha} f\right)(x)=\frac{(-1)^{n}}{\Gamma(n-\alpha)} \int_{x}^{b}(t-x)^{n-\alpha-1} f^{(n)}(t) d t, x<b .
$$

If $\alpha=n \in\{1,2,3, \ldots\}$ and usual derivative $f^{(n)}(x)$ of order $n$ exists, then Caputo fractional derivative $\left({ }^{c} D_{a^{+}}^{\alpha} f\right)(x)$ coincides with $f^{(n)}(x)$ whereas $\left({ }^{c} D_{b^{-}}^{\alpha} f\right)(x)$ coincides with $f^{(n)}(x)$ with exactness to a constant multiplier $(-1)^{n}$.

In particular for $n=1$ and $\alpha=0$, we have

$$
\left({ }^{c} D_{a^{+}}^{0} f\right)(x)=\left({ }^{c} D_{b^{-}}^{0} f\right)(x)=f(x) .
$$


Recently, Farid et al. [21] presented the following Hermite-Hadamard inequality for convex functions via Caputo fractional derivatives.

$$
\begin{gathered}
f^{(n)}\left(\frac{a+b}{2}\right) \leq \frac{\Gamma(n-\alpha+1)}{2(b-a)^{n-\alpha}}\left(\left({ }^{c} D_{a^{+}}^{\alpha} f\right)(b)+(-1)^{n}\left({ }^{c} D_{b^{-}}^{\alpha} f\right)(a)\right) \leq \\
\leq \frac{f^{(n)}(a)+f^{(n)}(b)}{2} .
\end{gathered}
$$

In the same paper, they established the following inequality for convex functions.

$$
\begin{aligned}
& \left|\frac{f^{(n)}(a)+f^{(n)}(b)}{2}-\frac{\Gamma(n-\alpha+1)}{2(b-a)^{n-\alpha}}\left[\left({ }^{c} D_{a^{+}}^{\alpha} f\right)(b)+(-1)^{n}\left({ }^{c} D_{(b)^{-}}^{\alpha} f\right)(a)\right]\right| \\
\leq & \frac{b-a}{2(n-\alpha+1)}\left(1-\frac{1}{2^{n-\alpha}}\right)\left(\left|f^{(n+1)}(a)\right|+\left|f^{(n+1)}(b)\right|\right) .
\end{aligned}
$$

Lemma 1. 22 For any $0 \leq a<b$ where $a, b \in \mathbb{R}$ and $0<\alpha \leq 1$, we have

$$
\left|a^{\alpha}-b^{\alpha}\right| \leq(b-a)^{\alpha} .
$$

Motivated by the above results, we present the analogue of Hermite-Hadamard inequality for preinvex functions via Caputo fractional derivatives. Also, we establish a new identity integral, and we derive some new trapezium type inequalities for preinvex functions via Caputo fractional derivatives. Finally, a briefly conclusion is provided as well.

\section{MAIN RESUltS}

Theorem 1. Let $f:[a, a+\eta(b, a)] \rightarrow \mathbb{R}$ be the function such that $f \in C^{n}$ $([a, a+\eta(b, a)])$ with $\eta(b, a)>0$. Assume that $f^{(n)}$ be positive and preinvex function on $[a, a+\eta(b, a)]$, and $\eta$ satisfies Condition $C$. Then the following inequalities for Caputo fractional derivatives hold:

$$
\begin{aligned}
& f^{(n)}\left(\frac{2 a+\eta(b, a)}{2}\right) \\
\leq & \frac{\Gamma(n-\alpha+1)}{2(\eta(b, a))^{n-\alpha}}\left(\left({ }^{c} D_{a^{+}}^{\alpha} f\right)(a+\eta(b, a))+(-1)^{n}\left({ }^{c} D_{(a+\eta(b, a))^{-}}^{\alpha} f\right)(a)\right) \\
\leq & \frac{f^{(n)}(a)+f^{(n)}(b)}{2} .
\end{aligned}
$$

Proof. Since $f^{(n)}$ is preinvex we have for every $x, y \in[a, a+\eta(b, a)]$

$$
f^{(n)}\left(x+\frac{1}{2} \eta(y, x)\right) \leq \frac{1}{2} f^{(n)}(x)+\frac{1}{2} f^{(n)}(y) .
$$

Putting $x=a+(1-t) \eta(b, a)$ and $y=a+t \eta(b, a)$ gives

$$
\begin{aligned}
& f^{(n)}\left(a+(1-t) \eta(b, a)+\frac{1}{2} \eta(a+t \eta(b, a), a+(1-t) \eta(b, a))\right) \\
\leq & \frac{1}{2}\left(f^{(n)}(a+(1-t) \eta(b, a))+f^{(n)}(a+t \eta(b, a))\right) .
\end{aligned}
$$


From Condition C, we have

$$
\begin{aligned}
& f^{(n)}\left(a+(1-t) \eta(b, a)+\frac{1}{2} \eta(a+t \eta(b, a), a+(1-t) \eta(b, a))\right) \\
= & f^{(n)}\left(a+(1-t) \eta(b, a)+\frac{2 t-1}{2} \eta(b, a)\right) \\
= & f^{(n)}\left(\frac{2 a+\eta(b, a)}{2}\right)
\end{aligned}
$$

Using 2.3) in 2.2), we obtain

$$
f^{(n)}\left(\frac{2 a+\eta(b, a)}{2}\right) \leq \frac{1}{2}\left(f^{(n)}(a+(1-t) \eta(b, a))+f^{(n)}(a+t \eta(b, a))\right) .
$$

Multiplying both sides of $(2.4)$ by $(n-\alpha) t^{n-\alpha-1}$ and integrating the resulting inequality with respect to $t$ over $[0,1]$, we get

$$
\begin{aligned}
& (n-\alpha) f^{(n)}\left(\frac{2 a+\eta(b, a)}{2}\right) \int_{0}^{1} t^{n-\alpha-1} d t \\
= & f^{(n)}\left(\frac{2 a+\eta(b, a)}{2}\right) \\
\leq & \frac{n-\alpha}{2} \int_{0}^{1} t^{n-\alpha-1} f^{(n)}(a+(1-t) \eta(b, a)) d t \\
& +\frac{n-\alpha}{2} \int_{0}^{1} t^{n-\alpha-1} f^{(n)}(a+t \eta(b, a)) d t \\
= & \frac{n-\alpha}{2(\eta(b, a))^{n-\alpha}} \int_{a}^{a+\eta(b, a)}(a+\eta(b, a)-u)^{n-\alpha-1} f^{(n)}(u) d u \\
& +\frac{n-\alpha}{2(\eta(b, a))^{n-\alpha}} \int_{a}^{a+\eta(b, a)}(u-a)^{n-\alpha-1} f^{(n)}(u) d u \\
= & \frac{\Gamma(n-\alpha+1)}{2(\eta(b, a))^{n-\alpha}}\left(\left({ }^{c} D_{a^{+}}^{\alpha} f\right)(a+\eta(b, a))+(-1)^{n}\left({ }^{c} D_{(a+\eta(b, a))^{-}}^{\alpha} f\right)(a)\right) .
\end{aligned}
$$

Hence,

$$
\begin{aligned}
& f^{(n)}\left(\frac{2 a+\eta(b, a)}{2}\right) \\
& \leq \frac{\Gamma(n-\alpha+1)\left(\left({ }^{c} D_{a^{+}}^{\alpha} f\right)(a+\eta(b, a))+(-1)^{n}\left({ }^{c} D_{(a+\eta(b, a))^{-}}^{\alpha} f\right)(a)\right)}{2(\eta(b, a))^{n-\alpha}} .
\end{aligned}
$$

Now, from the preinvexity of $f^{(n)}$, we have

$$
f^{(n)}(a+(1-t) \eta(b, a)) \leq t f^{(n)}(a)+(1-t) f^{(n)}(b)
$$


and

$$
f^{(n)}(a+t \eta(b, a)) \leq(1-t) f^{(n)}(a)+t f^{(n)}(b) .
$$

Summing 2.7 and 2.8, and multiplying the resulting by $\frac{n-\alpha}{2} t^{n-\alpha-1}$ and then integrating it with respect to $t$ over $[0,1]$, we get

$$
\begin{aligned}
& \frac{n-\alpha}{2}\left(\int_{0}^{1} t^{n-\alpha-1} f^{(n)}(a+(1-t) \eta(b, a)) d t+\int_{0}^{1} t^{n-\alpha-1} f^{(n)}(a+t \eta(b, a)) d t\right) \\
\leq & \frac{n-\alpha}{2}\left(f^{(n)}(a)+f^{(n)}(b)\right) \int_{0}^{1} t^{n-\alpha-1} d t,
\end{aligned}
$$

which implies

$$
\begin{aligned}
& \frac{\Gamma(n-\alpha+1)}{2(\eta(b, a))^{n-\alpha}}\left(\left({ }^{c} D_{a^{+}}^{\alpha} f\right)(a+\eta(b, a))+(-1)^{n}\left({ }^{c} D_{(a+\eta(b, a))^{-}}^{\alpha} f\right)(a)\right) \\
\leq & \frac{1}{2}\left(f^{(n)}(a)+f^{(n)}(b)\right) .
\end{aligned}
$$

The desired result follows from 2.6 and 2.9). The proof is completed.

Remark 2.1: Taking $\eta(b, a)=b-a$ in Theorem 1 we obtain ([21], Theorem 2.3).

Lemma 2. Let $f:[a, a+\eta(b, a)] \rightarrow \mathbb{R}$ be the function such that $f \in C^{n+1}$ $([a, a+\eta(b, a)])$ and $\eta(b, a)>0$. Then the following equality for Caputo fractional derivatives holds:

$$
\begin{aligned}
& \frac{f^{(n)}(a)+f^{(n)}(a+\eta(b, a))}{2} \\
& -\frac{\Gamma(n-\alpha+1)\left[\left({ }^{c} D_{a^{+}}^{\alpha} f\right)(a+\eta(b, a))+(-1)^{n}\left({ }^{c} D_{(a+\eta(b, a))^{-}}^{\alpha} f\right)(a)\right]}{2(\eta(b, a))^{n-\alpha}} \\
= & \frac{\eta(b, a)}{2} \int_{0}^{1}\left(t^{n-\alpha}-(1-t)^{n-\alpha}\right) f^{(n+1)}(a+t \eta(b, a)) d t .
\end{aligned}
$$

Proof. Let

and

$$
I_{1}=\int_{0}^{1} t^{n-\alpha} f^{(n+1)}(a+t \eta(b, a)) d t
$$

$$
I_{2}=\int_{0}^{1}(1-t)^{n-\alpha} f^{(n+1)}(a+t \eta(b, a)) d t .
$$

Integrating by parts $I_{1}$, we get

$$
\begin{aligned}
I_{1} & =\frac{1}{\eta(b, a)} f^{(n)}(a+\eta(b, a))-\frac{n-\alpha}{\eta(b, a)} \int_{0}^{1} t^{n-\alpha-1} f^{(n)}(a+t \eta(b, a)) d t \\
& =\frac{1}{\eta(b, a)} f^{(n)}(a+\eta(b, a))-\frac{n-\alpha}{(\eta(b, a))^{2}} \int_{a}^{a+\eta(b, a)}\left(\frac{u-a}{\eta(b, a)}\right)^{n-\alpha-1} f^{(n)}(u) d u \\
& =\frac{1}{\eta(b, a)} f^{(n)}(a+\eta(b, a))-\frac{(n-\alpha) \Gamma(n-\alpha)}{(\eta(b, a))^{n-\alpha+1}}(-1)^{n}\left({ }^{c} D_{(a+\eta(b, a))^{-}}^{\alpha} f\right)(a) .
\end{aligned}
$$


Similarly, we have

$$
\begin{aligned}
I_{2} & =-\frac{1}{\eta(b, a)} f^{(n)}(a)+\frac{n-\alpha}{\eta(b, a)} \int_{0}^{1}(1-t)^{n-\alpha-1} f^{(n)}(a+t \eta(b, a)) d t \\
& =-\frac{1}{\eta(b, a)} f^{(n)}(a)+\frac{n-\alpha}{(\eta(b, a))^{n-\alpha+1}} \int_{a}^{a+\eta(b, a)}(a+\eta(b, a)-u)^{n-\alpha-1} f^{(n)}(u) d u \\
& =-\frac{1}{\eta(b, a)} f^{(n)}(a)+\frac{(n-\alpha) \Gamma(n-\alpha)}{(\eta(b, a))^{n-\alpha+1}}\left({ }^{c} D_{a^{+}}^{\alpha} f\right)(a+\eta(b, a)) .
\end{aligned}
$$

Subtracting (2.11) from (2.10), and then multiplying the resulting equality by $\frac{\eta(b, a)}{2}$, we obtain the desired result.

Theorem 2. Let $f:[a, a+\eta(b, a)] \rightarrow \mathbb{R}$ be the function such that $f \in C^{n+1}$ $([a, a+\eta(b, a)])$ and $\eta(b, a)>0$. If $\left|f^{(n+1)}\right|$ is preinvex, then the following inequality for Caputo fractional derivatives holds:

$$
\begin{gathered}
\mid \frac{f^{(n)}(a)+f^{(n)}(a+\eta(b, a))}{2}- \\
-\frac{\Gamma(n-\alpha+1)\left[\left({ }^{c} D_{a^{+}}^{\alpha} f\right)(a+\eta(b, a))+(-1)^{n}\left({ }^{c} D_{(a+\eta(b, a))^{-}}^{\alpha} f\right)(a)\right]}{2(\eta(b, a))^{n-\alpha}} \mid \\
\leq \frac{\eta(b, a)}{2(n-\alpha+1)}\left(1-\left(\frac{1}{2}\right)^{n-\alpha}\right)\left(\left|f^{(n+1)}(a)\right|+\left|f^{(n+1)}(b)\right|\right) .
\end{gathered}
$$

Proof. From Lemma 2 properties of modulus and preinvexity of $\left|f^{(n+1)}\right|$, we have

$$
\begin{gathered}
\mid \frac{f^{(n)}(a)+f^{(n)}(a+\eta(b, a))}{2}- \\
\frac{\Gamma(n-\alpha+1)\left[\left({ }^{c} D_{a^{+}}^{\alpha} f\right)(a+\eta(b, a))+(-1)^{n}\left({ }^{c} D_{(a+\eta(b, a))^{-}}^{\alpha} f\right)(a)\right]}{2(\eta(b, a))^{n-\alpha}} \mid \\
\leq \frac{\eta(b, a)}{2} \int_{0}^{1}\left|t^{n-\alpha}-(1-t)^{n-\alpha}\right|\left|f^{(n+1)}(a+t \eta(b, a))\right| d t \\
=\frac{\eta(b, a)}{2}\left(\int_{0}^{\frac{1}{2}}\left((1-t)^{n-\alpha}-t^{n-\alpha}\right)\left|f^{(n+1)}(a+t \eta(b, a))\right| d t\right. \\
\left.+\int_{\frac{1}{2}}^{1}\left(t^{n-\alpha}-(1-t)^{n-\alpha}\right)\left|f^{(n+1)}(a+t \eta(b, a))\right| d t\right)
\end{gathered}
$$




$$
\begin{aligned}
& \leq \frac{\eta(b, a)}{2}\left(\int_{0}^{\frac{1}{2}}\left((1-t)^{n-\alpha}-t^{n-\alpha}\right)\left((1-t)\left|f^{(n+1)}(a)\right|+t\left|f^{(n+1)}(b)\right|\right) d t\right. \\
& \left.+\int_{\frac{1}{2}}^{1}\left(t^{n-\alpha}-(1-t)^{n-\alpha}\right)\left((1-t)\left|f^{(n+1)}(a)\right|+t\left|f^{(n+1)}(b)\right|\right) d t\right) \\
& =\frac{\eta(b, a)}{2}\left(| f ^ { ( n + 1 ) } ( a ) | \left(\int_{0}^{\frac{1}{2}}\left((1-t)^{n-\alpha+1}-t^{n-\alpha}(1-t)\right) d t\right.\right. \\
& \left.+\int_{\frac{1}{2}}^{1}\left(t^{n-\alpha}(1-t)-(1-t)^{n-\alpha+1}\right) d t\right) \\
& +\left|f^{(n+1)}(b)\right|\left(\int_{0}^{\frac{1}{2}}\left(t(1-t)^{n-\alpha}-t^{n-\alpha+1}\right) d t\right. \\
& \left.\left.+\int_{\frac{1}{2}}^{1}\left(t^{n-\alpha+1}-t(1-t)^{n-\alpha}\right) d t\right)\right) \\
& \left.+\int_{\frac{1}{2}}^{1}\left(t^{n-\alpha}(1-t)-(1-t)^{n-\alpha+1}\right) d t\right) \\
& =\frac{\eta(b, a)}{2(n-\alpha+1)}\left(1-\left(\frac{1}{2}\right)^{n-\alpha}\right)\left(\left|f^{(n+1)}(a)\right|+\left|f^{(n+1)}(b)\right|\right) \\
& \left(\left|f^{(n+1)}(a)\right|+\left|f^{(n+1)}(b)\right|\right)\left(\int_{0}^{\frac{1}{2}}\left((1-t)^{n-\alpha+1}-t^{n-\alpha}(1-t)\right) d t\right.
\end{aligned}
$$

where we have used the fact that

$$
\int_{0}^{\frac{1}{2}}\left((1-t)^{n-\alpha+1}-t^{n-\alpha}(1-t)\right) d t=\frac{1}{n-\alpha+2}-\frac{1}{n-\alpha+1}\left(\frac{1}{2}\right)^{n-\alpha+1}
$$

and

$$
\begin{gathered}
\int_{\frac{1}{2}}^{1}\left(t^{n-\alpha}(1-t)-(1-t)^{n-\alpha+1}\right) d t= \\
=\frac{1}{(n-\alpha+1)(n-\alpha+2)}-\frac{1}{n-\alpha+1}\left(\frac{1}{2}\right)^{n-\alpha+1} .
\end{gathered}
$$

The proof is completed.

Corollary 2.1. Choosing $\eta(b, a)=b-a$ in Theorem 2, we get (21], Theorem 2.4). 
Theorem 3. Let $f:[a, a+\eta(b, a)] \rightarrow \mathbb{R}$ be the function such that $f \in C^{n}$ $([a, a+\eta(b, a)])$ and $\eta(b, a)>0$. If $\left|f^{(n+1)}\right|^{q}$ is preinvex where $q>1$ with $\frac{1}{p}+\frac{1}{q}=1$, then the following inequality for Caputo fractional derivatives holds:

$$
\begin{gathered}
\mid \frac{f^{(n)}(a)+f^{(n)}(a+\eta(b, a))}{2}- \\
-\frac{\Gamma(n-\alpha+1)\left[\left({ }^{c} D_{a^{+}}^{\alpha} f\right)(a+\eta(b, a))+(-1)^{n}\left({ }^{c} D_{(a+\eta(b, a))^{-}}^{\alpha} f\right)(a)\right]}{2(\eta(b, a))^{n-\alpha}} \mid \\
\leq \frac{\eta(b, a)}{2}\left(\frac{1}{p(n-\alpha)+1}\right)^{\frac{1}{p}}\left(\frac{\left|f^{(n+1)}(a)\right|^{q}+\left|f^{(n+1)}(b)\right|^{q}}{2}\right)^{\frac{1}{q}} .
\end{gathered}
$$

Proof. From Lemmas 1 and 2, properties of modulus, Hölder's inequality and preinvexity of $\left|f^{(n+1)}\right|^{q}$, we have

$$
\begin{gathered}
\mid \frac{f^{(n)}(a)+f^{(n)}(a+\eta(b, a))}{2}- \\
-\frac{\Gamma(n-\alpha+1)\left[\left({ }^{c} D_{a^{+}}^{\alpha} f\right)(a+\eta(b, a))+(-1)^{n}\left({ }^{c} D_{(a+\eta(b, a))^{-}}^{\alpha} f\right)(a)\right]}{2(\eta(b, a))^{n-\alpha}} \mid \\
\leq \frac{\eta(b, a)}{2}\left(\int_{0}^{1}\left|\left(t^{n-\alpha}-(1-t)^{n-\alpha}\right)^{p}\right| d t\right)^{\frac{1}{p}}\left(\int_{0}^{1}\left|f^{(n+1)}(a+t \eta(b, a))\right|^{q} d t\right)^{\frac{1}{q}} \\
\leq \frac{\eta(b, a)}{2}\left(\int_{0}^{\frac{1}{2}}\left|\left(t^{n-\alpha}-(1-t)^{n-\alpha}\right)\right|^{p} d t+\int_{\frac{1}{2}}^{1}\left|\left(t^{n-\alpha}-(1-t)^{n-\alpha}\right)\right|^{p} d t\right)^{\frac{1}{p}} \\
\times\left(\int_{0}^{1}(1-t)\left|f^{(n+1)}(a)\right|^{q}+t\left|f^{(n+1)}(b)\right|^{q} d t\right)^{\frac{1}{q}} \\
\leq \frac{\eta(b, a)}{2}\left(\int_{0}^{\frac{1}{2}}(1-2 t)^{n p-\alpha p} d t+\int_{\frac{1}{2}}^{1}(2 t-1)^{n p-\alpha p} d t\right)^{\frac{1}{p}} \\
\times\left(\frac{\left|f^{(n+1)}(a)\right|^{q}+\left|f^{(n+1)}(b)\right|^{q}}{2}\right)^{\frac{1}{q}} \\
=\frac{\eta(b, a)}{2}\left(\frac{1}{p(n-\alpha)+1}\right)^{\frac{1}{p}}\left(\frac{\left|f^{(n+1)}(a)\right|^{q}+\left|f^{(n+1)}(b)\right|^{q}}{2}\right)^{\frac{1}{q}} .
\end{gathered}
$$

The proof is completed. 
Corollary 3.1. In Theorem 3, if we take $\eta(b, a)=b-a$, we obtain

$$
\begin{array}{r}
\left|\frac{f^{(n)}(a)+f^{(n)}(b)}{2}-\frac{\Gamma(n-\alpha+1)}{2(b-a)^{n-\alpha}}\left[\left({ }^{c} D_{a^{+}}^{\alpha} f\right)(b)+(-1)^{n}\left({ }^{c} D_{(b)^{-}}^{\alpha} f\right)(a)\right]\right| \leq \\
\leq \frac{b-a}{2}\left(\frac{1}{p(n-\alpha)+1}\right)^{\frac{1}{p}}\left(\frac{\left|f^{(n+1)}(a)\right|^{q}+\left|f^{(n+1)}(b)\right|^{q}}{2}\right)^{\frac{1}{q}} .
\end{array}
$$

Theorem 4. Let $f:[a, a+\eta(b, a)] \rightarrow \mathbb{R}$ be the function such that $f \in C^{n+1}$ $([a, a+\eta(b, a)])$ with $\eta(b, a)>0$. If $\left|f^{(n+1)}\right|^{q}$ is preinvex where $q \geq 1$, then the following inequality for Caputo fractional derivatives holds:

$$
\begin{gathered}
\mid \frac{f^{(n)}(a)+f^{(n)}(a+\eta(b, a))}{2}- \\
-\frac{\Gamma(n-\alpha+1)\left[\left({ }^{c} D_{a^{+}}^{\alpha} f\right)(a+\eta(b, a))+(-1)^{n}\left({ }^{c} D_{(a+\eta(b, a))^{-}}^{\alpha} f\right)(a)\right]}{2(\eta(b, a))^{n-\alpha}} \mid \\
\leq \frac{\eta(b, a)}{\sqrt[q]{2}(n-\alpha+1)}\left(1-\left(\frac{1}{2}\right)^{n-\alpha}\right)\left(\left|f^{(n+1)}(a)\right|^{q}+\left|f^{(n+1)}(b)\right|^{q}\right)^{\frac{1}{q}} .
\end{gathered}
$$

Proof. From Lemma 1 properties of modulus, power mean inequality and preinvexity of $\left|f^{(n+1)}\right|^{q}$, we have

$$
\begin{gathered}
\mid \frac{f^{(n)}(a)+f^{(n)}(a+\eta(b, a))}{2}- \\
-\frac{\Gamma(n-\alpha+1)\left[\left({ }^{c} D_{a^{+}}^{\alpha} f\right)(a+\eta(b, a))+(-1)^{n}\left({ }^{c} D_{(a+\eta(b, a))^{\alpha}}^{\alpha} f\right)(a)\right]}{2(\eta(b, a))^{n-\alpha}} \mid \\
\leq \frac{\eta(b, a)}{2}\left(\int_{0}^{1}\left|t^{n-\alpha}-(1-t)^{n-\alpha}\right| d t\right)^{1-\frac{1}{q}} \\
\times\left(\int_{0}^{1}\left|t^{n-\alpha}-(1-t)^{n-\alpha}\right|\left|f^{(n+1)}(a+t \eta(b, a))\right|^{q} d t\right)^{\frac{1}{q}} \\
\leq \frac{\eta(b, a)}{2}\left(\int_{0}^{\frac{1}{2}}\left((1-t)^{n-\alpha}-t^{n-\alpha}\right) d t+\int_{\frac{1}{2}}^{1}\left(t^{n-\alpha}-(1-t)^{n-\alpha}\right) d t\right)^{1-\frac{1}{q}} \\
\times\left(\int_{0}^{1}\left|t^{n-\alpha}-(1-t)^{n-\alpha}\right|\left((1-t)\left|f^{(n+1)}(a)\right|^{q}+t\left|f^{(n+1)}(b)\right|^{q}\right) d t\right)^{\frac{1}{q}} \\
=\frac{\eta(b, a)}{2}\left(\frac{2}{n-\alpha+1}\left(1-\left(\frac{1}{2}\right)^{n-\alpha}\right)\right)^{1-\frac{1}{q}}
\end{gathered}
$$




$$
\begin{gathered}
\times\left(| f ^ { ( n + 1 ) } ( a ) | ^ { q } \left(\int_{0}^{\frac{1}{2}}\left((1-t)^{n-\alpha+1}-t^{n-\alpha}(1-t)\right) d t\right.\right. \\
\left.+\int_{\frac{1}{2}}^{1}\left(t^{n-\alpha}(1-t)-(1-t)^{n-\alpha+1}\right) d t\right) \\
+\left|f^{(n+1)}(b)\right|^{q}\left(\int_{0}^{\frac{1}{2}}\left(t(1-t)^{n-\alpha}-t^{n-\alpha+1}\right) d t\right. \\
+\frac{\eta(b, a)}{2}\left(\frac{2}{n-\alpha+1}\left(1-\left(\frac{1}{2}\right)^{n-\alpha}\right)\right)^{1-\frac{1}{q}}\left(\left|f^{(n+\alpha+1)}(a)\right|^{q}+\left|f^{(n+1)}(b)\right|^{q}\right)^{\frac{1}{q}} \\
\times \frac{\eta(b, a)}{\sqrt{q}(n-\alpha+1)}\left(1-\left(\frac{1}{2}\right)^{n-\alpha}\right)\left(\left|f^{(n+1)}(a)\right|^{q}+\left|f^{(n+1)}(b)\right|^{q}\right)^{\frac{1}{q}} \\
\left.\left.\times\left(\int_{0}^{\frac{1}{2}}\left(t(1-t)^{n-\alpha}-t^{n-\alpha+1}\right) d t\right)\right)^{\frac{1}{q}}\left(t^{n-\alpha+1}-t(1-t)^{n-\alpha}\right) d t\right)^{\frac{1}{q}} \\
=\frac{\eta(b, a)}{2}\left(\frac{2}{n-\alpha+1}\left(1-\left(\frac{1}{2}\right)^{n-\alpha}\right) f^{1-\frac{1}{q}}\left(\left.\frac{1}{n+1)}(b)\right|^{q}\right)^{\frac{1}{q}}\right. \\
=
\end{gathered}
$$

The proof is completed.

Corollary 4.1. In Theorem 4, if we choose $\eta(b, a)=b-a$, we obtain

$$
\begin{aligned}
& \left|\frac{f^{(n)}(a)+f^{(n)}(b)}{2}-\frac{(n-\alpha) \Gamma(n-\alpha)}{2(b-a)^{n-\alpha}}\left[\left({ }^{c} D_{a^{+}}^{\alpha} f\right)(b)+(-1)^{n}\left({ }^{c} D_{(b)^{-}}^{\alpha} f\right)(a)\right]\right| \\
\leq & \frac{b-a}{2^{\frac{1}{q}}(n-\alpha+1)}\left(1-\left(\frac{1}{2}\right)^{n-\alpha}\right)\left(\left|f^{(n+1)}(a)\right|^{q}+\left|f^{(n+1)}(b)\right|^{q}\right)^{\frac{1}{q}} .
\end{aligned}
$$




\section{Conclusion}

In this paper, we presented the analogue of Hermite-Hadamard inequality for preinvex functions via Caputo fractional derivatives. Also, we established a new identity integral, and we derived some new trapezium type inequalities for preinvex functions via Caputo fractional derivatives. We hope that current work using our idea and technique will attract the attention of researchers working in mathematical analysis and other related fields in pure and applied sciences.

\section{REFERENCES}

[1] J. E. Pečarić, F. Proschan, Y. L. Tong, Convex functions, partial orderings, and statistical applications, Mathematics in Science and Engineering, 187. Academic Press, Inc., Boston, MA, 1992.

[2] J. Hadamard, Étude sur les propriétés des fonctions entières et en particulier d'une fonction considérée par Riemann, J. Math. Pures Appl., 58 (1893), 171-215.

[3] C. Hermite, Sur deux limites d'une intégrale définie, Mathesis, 3 (1883), 82.

[4] D. Baleanu, A. Kashuri, P. O. Mohammed, B. Meftah, General Raina fractional integral inequalities on coordinates of convex functions, Adv. Difference Equ., 2021 (2021), 82.

[5] T. S. Du, M. A. Awan, A. Kashuri, S. Zhao, Some $k$-fractional extensions of the trapezium inequalities through generalized relative semi- $(m, h)$-preinvexity, Appl. Anal., 100(3) (2021), $642-662$.

[6] A. Kashuri, B. Meftah, P. O. Mohammed, Some weighted Simpson type inequalities for differentiable s-convex functions and their applications, J. Frac. Calc. \& Nonlinear Sys., 1(1) (2021), 75-94.

[7] M. A. Khan, Y. M. Chu, A. Kashuri, R. Liko, G. Ali, Conformable fractional integrals versions of Hermite-Hadamard inequalities and their generalizations, J. Funct. Spaces, 2018 (2018), Art. ID 6928130, 9 pp.

[8] B. Meftah, M. Merad, A. Souahi, Some Hermite-Hadamard type inequalities for functions whose derivatives are quasi-convex, Jordan J. Math. Stat., 12(2) (2019), 219-231.

[9] B. Meftah, M. Merad, N. Ouanas, A. Souahi, Some new Hermite-Hadamard type inequalities for functions whose $n^{\text {th }}$ derivatives are convex, Acta Comment. Univ. Tartu. Math., 23(2) (2019), 163-178.

[10] B. Meftah, New integral inequalities through the $\phi$-preinvexity, Iran. J. Math. Sci. Inform., 15(1) (2020), 79-83.

[11] B. Meftah, K. Mekalfa, Some weighted trapezoidal type inequalities via h-preinvexity, Rad Hrvat. Akad. Znan. Umjet. Mat. Znan., 24 (2020), 81-97.

[12] B. Meftah, A. Kashuri, Some new type integral inequalities for approximately harmonic $h-$ convex functions, Mat. Bilten., 44(1) (2020), 13-36.

[13] B. Meftah, M. Benssaad, W. Kaidouchi, S. Ghomrani, Conformable fractional HermiteHadamard type inequalities for product of two harmonic s-convex functions, Proc. Amer. Math. Soc., 149 (2021), 1495-1506.

[14] S. S. Dragomir, R. P. Agarwal, Two inequalities for differentiable mappings and applications to special means of real numbers and to trapezoidal formula, Appl. Math. Lett., 11(5) (1998), 91-95.

[15] W. W. Breckner, Stetigkeitsaussagen für eine Klasse verallgemeinerter konvexer Funktionen in topologischen linearen Räumen, (German), Publ. Inst. Math. (Beograd) (N.S.), 23(37) (1978), 13-20.

[16] T. Weir, B. Mond, Pre-invex functions in multiple objective optimization, J. Math. Anal. Appl., 136(1) (1988), 29-38. 
[17] S. R. Mohan, S. K. Neogy, On invex sets and preinvex functions, J. Math. Anal. Appl., 189(3) (1995), 901-908.

[18] M. A. Noor, Hermite-Hadamard integral inequalities for log-preinvex functions, J. Math. Anal. Approx. Theory, 2(2) (2007), 126-131.

[19] A. Barani, A. G. Ghazanfari, S. S. Dragomir, Hermite-Hadamard inequality for functions whose derivatives absolute values are preinvex, J. Inequal. Appl., 2012 (2012), 247, 9 pp.

[20] A. A. Kilbas, H. M. Srivastava, J. J. Trujillo, Theory and applications of fractional differential equations, North-Holland Mathematics Studies, 204. Elsevier Science B.V., Amsterdam, 2006.

[21] G. Farid, A. Javed, S. Naqvi, Hadamard and Fejér-Hadamard inequalities and related results via Caputo fractional derivatives, Bull. Math. Anal. Appl., 9(3) (2017), 16-30.

[22] J. Wang, C. Zhu, Y. Zhou, New generalized Hermite-Hadamard type inequalities and applications to special means, J. Inequal. Appl., 2013 (2013), 325, 15 pp.

1 University of 8 May 1945 Guelma, LABoratoire des téléCOMMUNiCATIONS, Faculté des Sciences et de la Technologie, P.O. Box 401, 24000 Guelma, Algeria

Email address: badrimeftah@yahoo.fr

2 University "Ismail Qemali", Department of Mathematics, Faculty of Technical Science, 9400 Vlora, Albania

Email address: artionkashuri@gmail.com

Received: 16.4 .2021

Accepted: 3.8 .2021 